\title{
Acylation of Blueberry Anthocyanins with Aliphatic Carbonyl Acids and Their Stability Analysis
} \author{
Qing Xu${ }^{1}$, Yuanjing Zhou ${ }^{2}$, Liping Luo ${ }^{1}$, Zhu Huang ${ }^{1}$, Fei Nie ${ }^{1}$ and Guilong
Gao $^{1, *}$ \\ ${ }^{1}$ Institute of Biology, Guizhou Academy of Sciences, Guiyang 550009, China \\ ${ }^{2}$ Institute of Analysis and Testing, Guizhou Academy of Sciences, Guiyang 550002, China \\ *email: xqhaoy@163.com
}

Keywords: Blueberry anthocyanins; aliphatic carbonyl acids; acylation; stability

Abstract: The instability of natural anthocyanins was a big obstacle for its usage. In order to improve the stability of anthocyanins, molecular modification was used. In this paper, the molecular modification of blueberry anthocyanins was acylated by using aliphatic carbonyl acids (acetic acid, oxalic acid, heptane acid, and lauric acid). The effects of acylation and the acylated agent with different length of carbon chain on stability of blueberry anthocyanins were studied. The infrared spectrum showed that the blueberry anthocyanins had been acylated. The stabilities (light and heat) were significantly increased being compared with no acylated anthocyanins. Results showed that the acylation method could increase the stability of original anthocyanin extracts.

\section{Introduction}

Anthocyanins, as highly colored substances found in plants, are possible viable colorants for using in food, nutraceutical, and pharmaceutical preparations. However, natural pigment exists some defects, especially its poor stability, which is affected by $\mathrm{pH}$, oxidation, light, temperature and so on. In the process of processing and circulation, it is vulnerable to external influence and can be easily degraded. Therefore, improving the stability of pigment is the key to promote the development and application of natural pigment. Some studies reported that the acylation of natural pigment has high stability ${ }^{[1-3]}$. For example, Fossen et al. found that petanin with one aromatic acyl group from blue potatoes (Solanum tuberosum var. Congo) showed both higher colour intensity and higher stability than cyanidin 3-O- $\beta$-D-glucopyranoside from natural rice (Oriza sativa L.) at high $\mathrm{pH}^{[1]}$. Sadilova et al. demonstrated that the acylated anthocyanins of black carrot were more stable, and exhibited higher half-life value than the nonacylated anthocyanins at high temperature ${ }^{[2]}$. Yawadio and Morita proved that the color of black rice anthocyanin-rich fraction enhanced by adding carboxylic acids into the anthocyanin-glucosides medium ${ }^{[3]}$. At present, the acylation of anthocyanins is mainly from the separation of natural products, and the use of chemical methods to synthesize acylated anthocyanins is rarely reported. Bluebery (or bilberry), also known as Vaccinium vitisidaea L., is rich in anthocyanidins, which have a variety of biological activities and health benefits, and have widespread applications in the fields of drugs, cosmetics, food additives and so on ${ }^{[4,5]}$. However, the instability of the blueberry anthocyanin is seriously restricting its application. In this paper, by the method of chemical synthesis to introduce the aliphatic carbonyl acids to blueberry anthocyanin was carried out, and the effects of acylation and the acylated agent with different length of carbon chain on stability of blueberry anthocyanins were investigated. The results would help further understand the relationship between the stability of anthocyanin and structure, and expand the application of blueberry anthocyanins.

\section{Materials and methods}

Materials and Reagents Fresh blueberries (Vaccinium ashei) were harvested from blueberry planting base in Majiang County, Guizhou Province, China, in August, 2016. All chemicals used were 
of the highest grades available. Deionized water was prepared from a Milli-Q Element water purification system (Millipore Co., Billerica, MA, USA).

Chemical Acylation Procedure Blueberry anthocyanin was dissolved in 30\% methanol aqueous solution, and the solution was adjusted to $\mathrm{pH} 6$ by dilute $\mathrm{NaOH}$ aqueous solution. Then, carboxylic acid (acetic acid, oxalic acid, heptane acid, or lauric acid) was added into the solution. The reaction was protected by nitrogen and continuously stirred at $60^{\circ} \mathrm{C}$. Samples were obtained every $1 \mathrm{~h}$, diluted with methanol, and analyzed by thin-layer chromatography (TLC) to monitor the extent of reaction. When the reaction was completed, it was stopped and cooled to room temperature. The reaction solution was filtrated. The filtrate was concentrated by vacuum and dried by sublimation.

FTIR Analysis Fourier transform infrared spectroscopy (FTIR) tests were conducted on a Spectrum 100 FTIR spectrometer (PerkinElmer, US). The spectral region was 4000 to $400 \mathrm{~cm}^{-1}$ and the resolution ratio was $2 \mathrm{~cm}^{-1}$. Upon scanning, interference of $\mathrm{H}_{2} \mathrm{O}$ and $\mathrm{CO}_{2}$ was excluded. Blueberry anthocyanin and acylated blueberry anthocyanin were separately combined with $\mathrm{KBr}$ by grinding evenly.

Stability Analysis Dual-wavelength $\mathrm{pH}$ differential method was used to figure out the retention rate, using UV detector to detect. Take $1 \mathrm{~mL}$ diluted sample, add $9 \mathrm{~mL}$ buffer solution which $\mathrm{pH}$ is 1.0 and 4.5 respectively, and then detect the absorbance in 510 and $700 \mathrm{~nm}$ after a water bath for $40 \mathrm{~min}$. The retention rate of blueberry anthocyanin was calculated by the following formula:

$$
\text { Retention rate }(\%)=\mathrm{A}_{2} / \mathrm{A}_{1} \times 100
$$

Statistical Analysis Experiments were performed at repeating three times, and the results were expressed as means standard deviation. Statistical analysis was performed using SPSS 16.0. Differences between groups were considered significant at $\mathrm{P}<0.05$.

\section{Results and discussion}

FTIR Analysis The FTIR spectrograms of blueberry anthocyanin and acylated blueberry anthocyanin are shown in Fig.1.

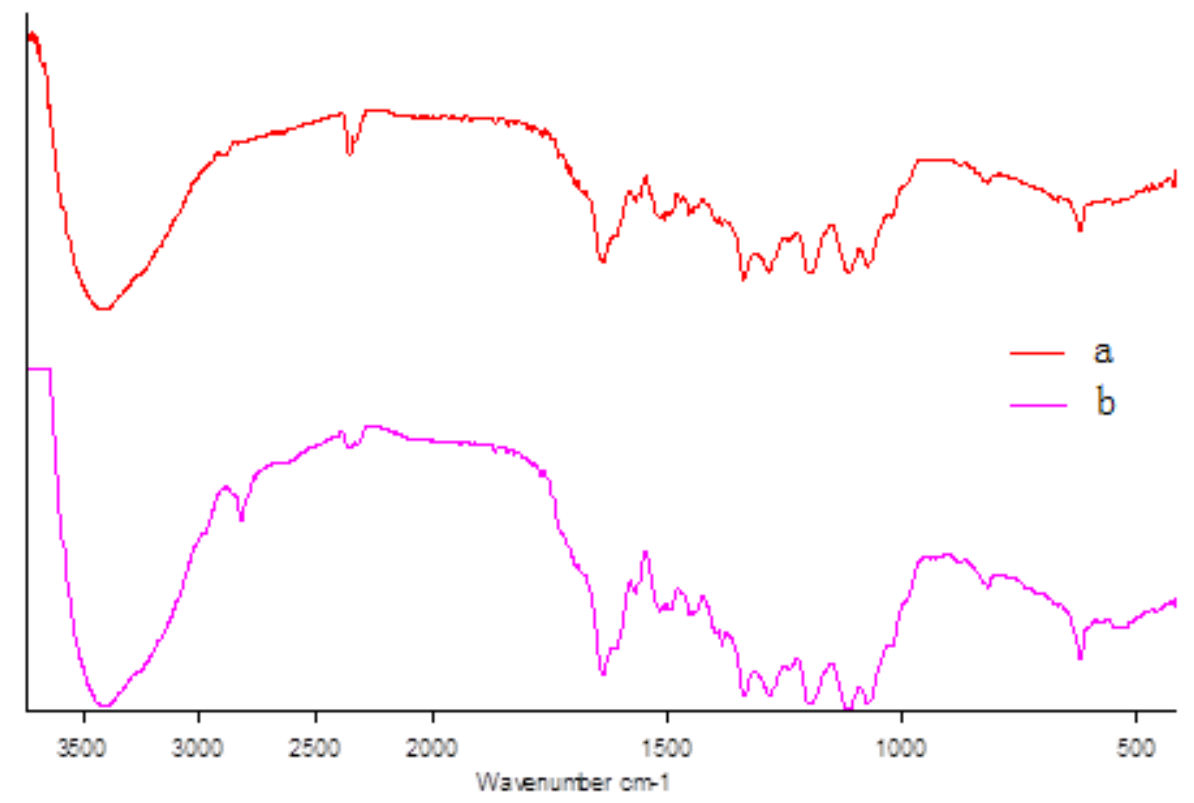

Figure 1 The line of a was the FTIR spectrum of blueberry anthocyanin, the line of $b$ was the FTIR spectrum of acylated blueberry anthocyanin.

According to the location and shape of the peak, the wide and strong absorption peak at about 3425 $\mathrm{cm}^{-1}$ corresponded to many hydroxide radical stretching vibration peaks of aromatic ring and glycosyl in the structure of before and after acylation of blueberry anthocyanin. The absorption peaks at around 1640, 1600, 1508 and $1456 \mathrm{~cm}^{-1}$ corresponded to the aromatic and heterocyclic rings' skeletal vibration in the chromene of before and after acylation of blueberry anthocyanin. A series of absorption peaks between 1338 to $1024 \mathrm{~cm}^{-1}$ corresponded to the stretching vibration of $\mathrm{C}-\mathrm{O}$ in glycosyl. 
Compared with the blueberry anthocyanin, an obviously high absorption peak at $2930 \mathrm{~cm}^{-1}$ belonged to the saturated hydrocarbon stretching vibrations, and the high absorption peak at around $1255 \mathrm{~cm}^{-1}$ corresponded to the stretching vibration of $\mathrm{C}-\mathrm{O}-\mathrm{C}$ perssad. These could be the primary characteristic of ester groups' existence. Therefore, blueberry anthocyanin was acylated by aliphatic carbonyl acids.

\section{Stability Analysis}

Fig. 2 showed the retention rates of blueberry anthocyanin and acylated blueberry anthocyanins with increasing temperature and extension of heating time. All of the acylated blueberry anthocyanins $(\mathbf{2}, \mathbf{3}, \mathbf{4}$, and $\mathbf{5})$ were found to possess higher heat resistance than the blueberry anthocyanin $(\mathbf{1})$. When the heating time was longer than $4 \mathrm{~h}$, the retention rate of acylated blueberry anthocyanin was obviously higher than that of blueberry anthocyanins. At 80 and $100{ }^{\circ} \mathrm{C}$, the retention rate of blueberry anthocyanin were 50.18 and $22.27 \%$, respectively, and the acylated blueberry anthocyanins could be up to 70.24 and $60.47 \%$, respectively. With increasing temperature, the effect of heat resistance became more obvious. Blueberry anthocyanin has free hydroxyl groups in the cyclo-chromene and in the 6'glucoside. Thus, the hydroxyl groups make blueberry anthocyanin unstable. Acylation of the ester can improve blueberry anthocyanin's stability. The ester group was more stable than hydroxide radicals, which is in agreement with the results reported by Sadilova ${ }^{[2]}$.
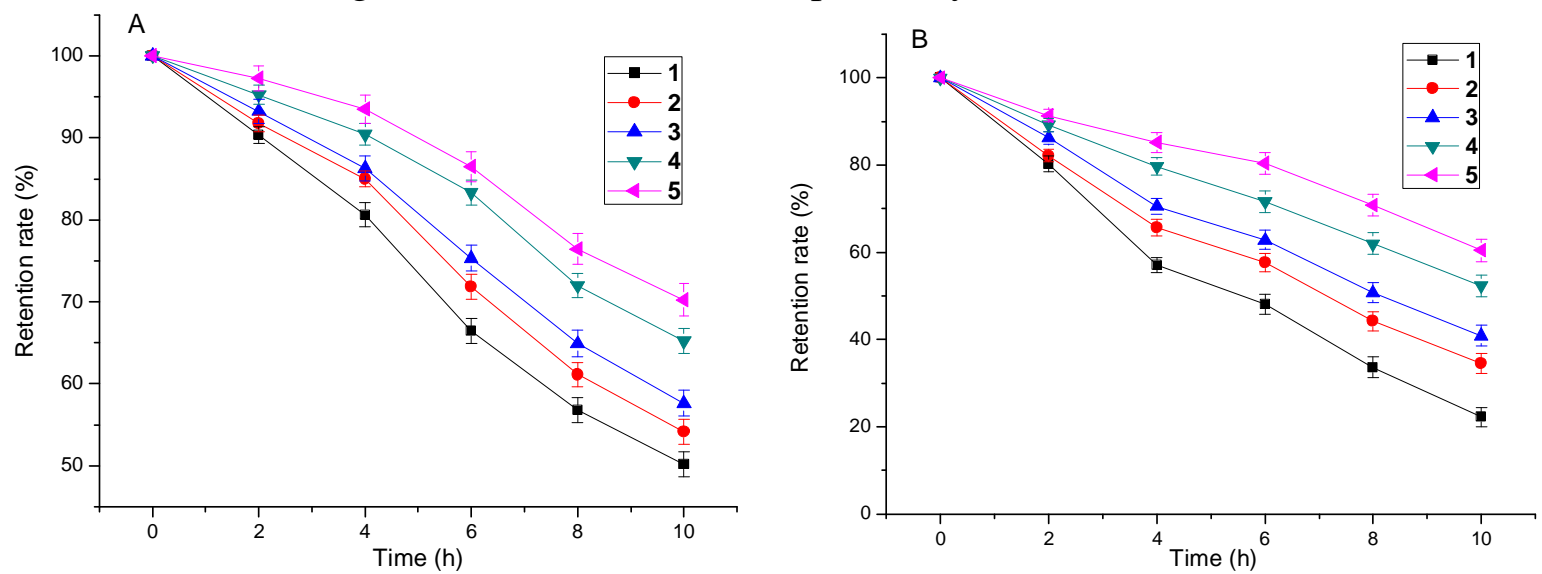

Figure 2 The effect of different temperatures on stability of blueberry anthocyanin and acylated blueberry anthocyanin. A: temperature at $80^{\circ} \mathrm{C}$; B: temperature at $100^{\circ} \mathrm{C}$. 1: blueberry anthocyanin; 2: acetic acid acylated blueberry anthocyanin; 3: oxalic acid acylated blueberry anthocyanin; 4: heptane acid acylated blueberry anthocyanin; 5: lauric acid acylated blueberry anthocyanin.
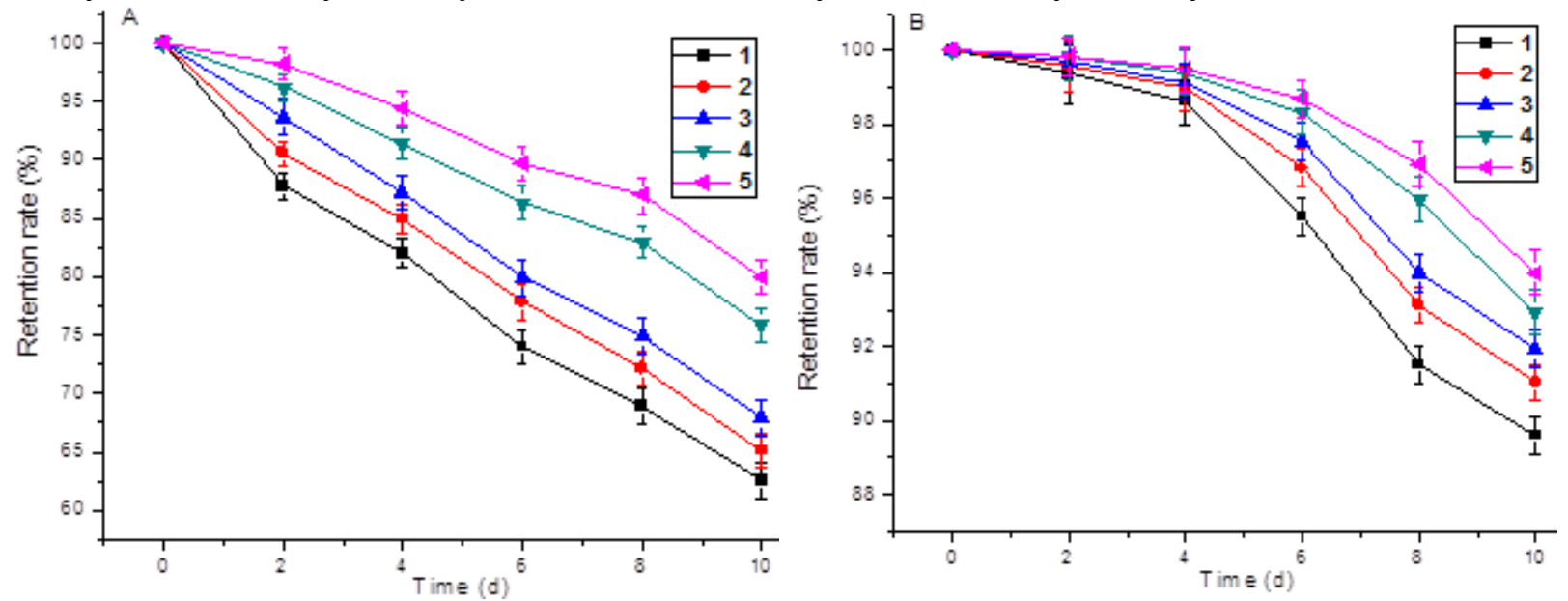

Figure 3 The effect of different lighting on stability of blueberry anthocyanin and acylated blueberry anthocyanin. A: indoor natural light; B: indoor avoiding light. 1-4 are the same as Figure 2.

Fig. 3 showed the stability of blueberry anthocyanin and acylated blueberry anthocyanins on different lights with extension of lighting time. Compared with the control substance placed in indoor avoiding light place (Fig. 3B), blueberry anthocyanin and acylated blueberry anthocyanins were exposed to indoor sunlight for a period of time (Fig. 3A), and the retention rate decreased rapidly. This indicated that blueberry anthocyanin had poor stability to light and need to be protected from light. As 
shown in Fig. 3A, with prolonged lighting time, the stability of blueberry anthocyanin and acylated blueberry anthocyanins decreased. The stability of acylated blueberry anthocyanins was higher than that of blueberry anthocyanin. When lighting was extended for $10 \mathrm{~d}$, the retention rate of acylated blueberry anthocyanins remained higher than $65 \%$, whereas, that of blueberry anthocyanin was $60 \%$.

As can be seen from Fig. 2 and Fig. 3, the stability of acylated blueberry anthocyanins $(\mathbf{2}, \mathbf{3}, \mathbf{4}$, and 5) on temperature and light increased with the increase of carbon chain of aliphatic carbonyl acid. When the carbon chain increased to 8 (4 vs $\mathbf{5}$ ), the stability of acylated blueberry anthocyanin on temperature and light changed a little.

\section{Conclusions}

The acylated blueberry anthocyanin had higher stability than blueberry anthocyanin at different temperatures and lighting times. The stability of acylated blueberry anthocyanin on temperature and light increased with the increase of carbon chain of aliphatic carbonyl acid. Acylated blueberry anthocyanins with high stability can expand their application in food, nutraceutical, and pharmaceutical industry.

\section{Acknowledgements}

This work was financially supported by the Youth Fund Project of Guizhou Academy of Sciences, Guizhou province, China (J [2016]20), Science and Technology Project of Guizhou Province, China (JZ [2015]2006, Z [2015]6013, [2016]1139), and National Natural Science Foundation (81560603).

\section{References}

[1] T. Fossen, L Cabrita and O.M. Andersen: Food Chem., 63 (1998), 435-440.

[2] E. Sadilova, F.C. Stintzing and R. Carle: J. Food Sci., 71 (2006), C504-512.

[3] R. Yawadio and N. Morita: Food Chem., 105 (2007), 421-427.

[4] F.S. Hosseinian, and T. Beta: J. Agr. Food Chem., 55 (2007), 10832-10838.

[5] Q. Xu, Y. Zhou, Y. Wu, et al.: Proceedings of the 2016 International Conference on Biotechnology \& Medical Science, 2016, 104-109. 